\title{
Does Open Reduction and Internal Fixation versus Primary Arthrodesis Improve Patient Outcomes for Lisfranc Trauma? A Systematic Review and Meta-analysis
}

\author{
Nicholas Smith MD, MSc, Craig Stone MD, MSc, FRCSC, \\ Andrew Furey MD, MSc, FRCSC
}

Published online: 29 May 2015

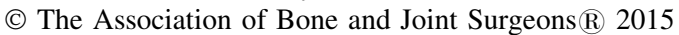

\begin{abstract}
Background Although Lisfranc injuries are uncommon, representing approximately $0.2 \%$ of all fractures, they are complex and can result in persistent pain, degenerative arthritis, and loss of function. Both open reduction and internal fixation (ORIF) and primary fusion have been proposed as treatment options for these injuries, but debate remains as to which approach is better.

Questions/purposes We asked whether ORIF or primary fusion led to (1) fewer reoperations for hardware removal;

(2) less frequent revision surgery; (3) higher patient outcome scores; and (4) more frequent anatomic reduction.

Methods A systematic review was performed using the Preferred Reporting Items for Systematic Reviews and Meta-Analysis (PRISMA) guidelines. Three trials met the criteria for inclusion within the meta-analysis. Qualifying
\end{abstract}

Each author certifies that he or she, or a member of his or her immediate family, has no funding or commercial associations (eg, consultancies, stock ownership, equity interest, patent/licensing arrangements, etc) that might pose a conflict of interest in connection with the submitted article.

All ICMJE Conflict of Interest Forms for authors and Clinical Orthopaedics and Related Research ${ }^{\circledR}$ editors and board members are on file with the publication and can be viewed on request.

Clinical Orthopaedics and Related Research ${ }^{\mathbb{R}}$ neither advocates nor endorses the use of any treatment, drug, or device. Readers are encouraged to always seek additional information, including FDAapproval status, of any drug or device prior to clinical use.

N. Smith

Memorial University of Newfoundland, St John's, NL, Canada

C. Stone

General Orthopaedics/Foot and Ankle Surgery, Department of Orthopaedic Surgery, Discipline of Surgery, Faculty of Medicine, Memorial University of Newfoundland, St John's, NL, Canada articles for the meta-analysis had data extracted independently by two authors (NS, AF). The quality of each study was assessed using the Center for Evidence Based Medicine's evaluation strategy; data were extracted from articles rated as good and fair: two and one article, respectively.

Results The risk ratio for hardware removal was 0.23 (95\% confidence interval [CI], 0.11-0.45; $\mathrm{p}<0.001$ ) indicating more hardware removal for ORIF than fusion. For other revision surgery, the risk ratio for ORIF was 0.36 (95\% CI, 0.08-1.59; $\mathrm{p}=0.18$ ) favoring neither. Similarly, neither was favored using patient-reported outcomes; the standard mean difference was calculated to be 0.50 (95\% $\mathrm{CI},-2.13$ to $3.12 ; \mathrm{p}=0.71)$. When considering the risk of nonanatomic alignment, neither was favored (risk ratio, 1.48 ; 95\% CI, 0.34-6.38; $\mathrm{p}=0.60$ ).

Conclusions The surgeon should consider the increased risk of hardware removal along with its associated morbidity and discuss this with the patient preoperatively when considering ORIF of Lisfranc injuries. Because no new trials have been performed since 2012, further randomized controlled trials will be needed improve our understanding of these interventions.

Level of Evidence Level I, therapeutic study.

\footnotetext{
A. Furey ( $\square)$

Orthopaedic Traumatology, Department of Surgery, Discipline of Surgery, Faculty of Medicine, Memorial University of Newfoundland, Room 1380, Health Science Center, 300 Prince Philip Drive, St John's, NL A1B3V6, Canada e-mail: andrewfurey@hotmail.com
} 


\section{Introduction}

The Lisfranc ligament runs on the plantar aspect of the foot from the lateral aspect of the medial cuneiform to the medial aspect of the second metatarsal [6]. It functions to stabilize the tarsometatarsal articulation of the foot and injury to the ligament can lead to substantial pain, midfoot arthritis, decreased function, and loss of quality of life [4]. This is a relatively rare injury with an incidence of approximately $0.2 \%$ of all fractures [1] although it is frequently diagnosed late and this leads to poor functional outcomes for the patient [5]. It has been demonstrated that anatomic reduction and rigid stabilization of the Lisfranc complex is the standard of care for these injuries [27]. Open reduction and internal fixation (ORIF) and primary arthrodesis have both been evaluated as treatment options with acceptable results [18].

Debate remains over which approach, ORIF or primary fusion, leads to better function and less pain. A systematic review in 2012 included data from six studies, compared American Orthopaedic Foot and Ankle Society Scores (AOFAS) and the percentage of patients achieving anatomic reduction between the ORIF and primary arthrodesis groups, and concluded that both methods were acceptable. The quality of evidence was low and more direct comparative trials would be needed [25]. However, their search criteria were not clearly defined and they did not perform a methodologically strong systematic review. A 2012 systematic review of three trials (two randomized controlled trials [RCTs] and one comparative series) likewise concluded that neither approach was superior based on the available literature and further trials would be needed to improve the evidence [10], but that study did not include an in-depth examination of the gray literature and non-English language articles were excluded. They recommended that further RCTs be performed to improve the outcome knowledge of these procedures. We sought to update the currently available literature with a clearly defined systematic review and pool the results in a meta-analysis. The Preferred Reporting Items for Systematic Reviews and MetaAnalysis (PRISMA) guidelines were adhered to throughout the study [15]. The purpose of this systematic review is to determine if patients who sustain acute traumatic Lisfranc injuries, either purely ligamentous or bony, achieve improved patient outcomes with primary fusion compared with ORIF in terms of (1) hardware removal; (2) revision surgery; (3) patient outcome measures; and (4) anatomic reduction.

\section{Search Strategy and Criteria}

\section{Eligibility Criteria}

RCTs and cohort studies comparing ORIF with primary arthrodesis were included in the evaluation. All human, comparative studies were included. Non-English studies were examined and translated if appropriate and possible.

\section{Search Strategy and Information Sources}

We searched the PubMed, EMBASE, and Cochrane databases in October 2014. No restrictions were placed on the search engines. Our initial search was performed in PubMed using terms to describe the anatomic location, injury type, and intervention. Lisfranc injuries were captured using the terms: "metatarsal bones"[Mesh], Lisfranc [tiab], midfoot [tiab], "tarsal joints" [Mesh], and "tarsal bone$\mathrm{s}$ "[Mesh]. Injury type was found using the keyword search "fracture" and the Mesh term "dislocations". Finally, surgical intervention was found using the terms: fixation [tiab], "fracture fixation"[Mesh], "fracture fixation, internal" [Mesh], fusion [tiab], and "arthrodesis"[Mesh]. All possible combinations of the terms were applied.

This strategy produced 318 papers in PubMed. A similar protocol was undertaken in EMBASE and the Cochrane Library revealing 701 and 263 papers, respectively. Reference lists from relevant articles were examined for further studies. Clinical trial databases from the World Health Organization, Health Canada, and the US National Institutes of Health were explored for registered trials. Only one clinical trial has been registered and although it is actively recruiting patients, it has yet to produce clinical outcomes.

An online search engine, www.duckduckgo.com, was used to examine the gray literature with a similar search strategy as that used for examining PubMed. Web sites for the American Academy of Orthopaedic Surgeons, the AOFAS, and the Canadian Orthopaedic Association were reviewed for recent meetings. Conference proceedings and unpublished data were sought. Two foot and ankle specialists were contacted as content experts to identify current studies being completed in North America. The total number of papers found through the database search was 1282 with an additional 15 articles discovered through the other sources. This was felt to be broad enough to identify all potentially related work (Fig. 1). Duplicates were removed and titles were scanned for initial screening. Foreign language articles with English abstract translations were accepted. Articles were excluded for basic science work, nonhuman trials, surgical technique guides, pediatric trials, other bony and ligamentous injuries, commentaries, meeting summaries, biomechanical studies, and textbook chapters. This left 39 articles for screening of their entire texts. Papers were excluded if they were reviews, had no comparative data, or dealt with neuropathic joints. Translations were performed for one French and one German-language article, which left nine papers for in-depth evaluation. 


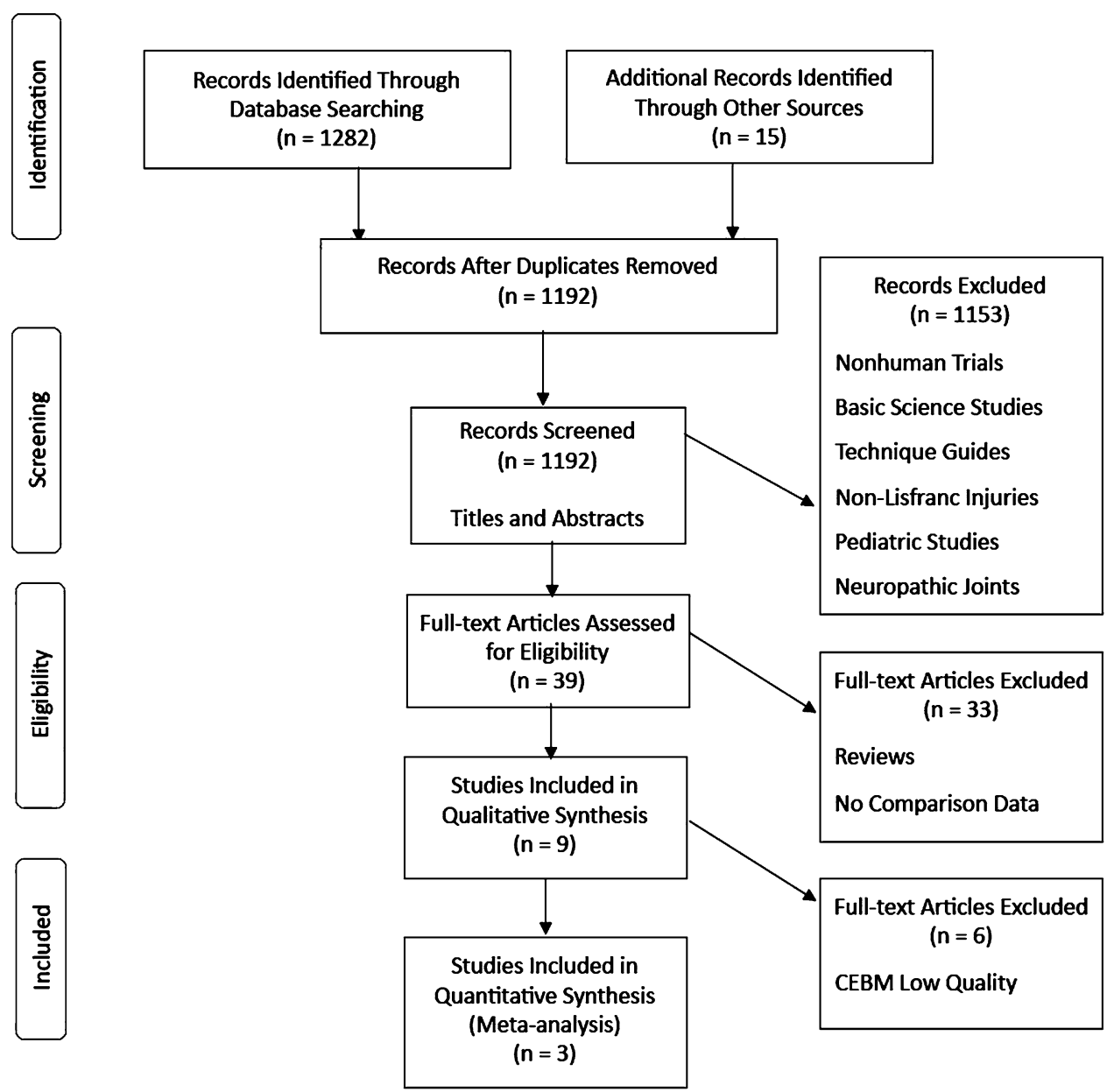

Fig. 1 PRISMA flow diagram compares ORIF with primary fusion for Lisfranc injuries.

\section{Study Selection and Data Collection Process}

The Center for Evidence-Based Medicine's [3] strategy for quality assessment of scientific literature was used to critique each paper (Table 1). Only RCTs and high-quality cohort studies were included in the analysis. The US Preventive Task Force Quality Rating Criteria were used to evaluate for risk of bias (Table 2). Acceptable articles contained data comparing ORIF with primary arthrodesis and were rated as "good" or "fair" on the quality rating scale. Potential sources of bias came from minor loss to followup, problems with statistical power, and heterogeneity within injury patterns and treatment protocols. Results extracted were patient outcome scores, need for a revision, removal of hardware, and quality of reduction radiographically. The two primary researchers (AF, NS) examined each of the articles independently and the senior author (CS) resolved any disagreement. Attempts were made to contact authors when outcome measures were not reported. Statistical analysis was completed using Review Manager software (Version 5.3.5; The Nordic Cochrane Center, The Cochrane Collaboration, Copenhagen, Denmark).
For hardware removal risk, we assessed differences in proportions having undergone hardware removal. We included two studies that reported on this outcome variable. For repeat surgery, we evaluated risk of return to the operating room for reasons other than hardware removal. All three studies discuss the reasons for repeat surgery including: hardware failure, posttraumatic arthritis, and loss of reduction. For patient-reported outcomes, Henning used the Short Musculoskeletal Functional Assessment and the SF-36. Incomplete statistical sets were available for a meta-analysis and this study could not be included. Complete statistical data were available for Ly et al. [13], who used the AOFAS scale, and for Mulier who used the Baltimore Painful Foot score. Different scales were present; therefore, we assessed standard mean differences.

We also evaluated the risk ratio of nonanatomic alignment. Proportions of anatomic reduction radiographically were compared using risk ratios for the two techniques. All three studies reported on this measure. Heterogeneity was assessed using the $\mathrm{I}^{2}$ measure. Random-effects models were used for all statistical calculations given the heterogeneity noted between the studies along with the Mantel- 
Table 1. Summary of articles in this meta-analysis

\begin{tabular}{llllll}
\hline Study & Design & Number of patients & Treatment group & Control group & Outcomes measured \\
\hline Henning et al., 2009 [8] & RCT & 32 & Fusion & ORIF & SF-36, SMFA, radiography, secondary surgery \\
Ly et al., 2006 [13] & RCT & 41 & Fusion & ORIF & Radiography, secondary surgery, AOFAS \\
Mulier et al., 2002 [16] & RC & 28 & Fusion & ORIF & BPFS, radiography, satisfaction \\
\hline
\end{tabular}

$\mathrm{RC}=$ retrospective cohort; $\mathrm{RCT}=$ randomized controlled trial; ORIF $=$ open reduction and internal fixation; SMFA = Short Musculoskeletal Functional Analysis scores; AOFAS = American Orthopaedic Foot and Ankle Society painful foot scale; BPFS = Baltimore Painful Foot System .

Table 2. Assessment of potential bias of studies selection for inclusion in the synthesis using USPSTF Quality Rating Criteria

\begin{tabular}{llllllll}
\hline Study & $\begin{array}{l}\text { Assembly } \\
\text { of comparable } \\
\text { groups }\end{array}$ & $\begin{array}{l}\text { Maintenance } \\
\text { of comparable } \\
\text { groups }\end{array}$ & $\begin{array}{l}\text { No important } \\
\text { differential loss } \\
\text { to follow-up or } \\
\text { overall high loss } \\
\text { to follow-up }\end{array}$ & $\begin{array}{l}\text { Measurements: } \\
\text { equal, reliable, } \\
\text { valid (includes } \\
\text { masking of } \\
\text { outcome } \\
\text { assessment) }\end{array}$ & $\begin{array}{l}\text { Clear } \\
\text { definition of } \\
\text { interventions }\end{array}$ & $\begin{array}{l}\text { All } \\
\text { important } \\
\text { outcomes } \\
\text { considered }\end{array}$ & $\begin{array}{l}\text { Analysis: } \\
\text { adjustment } \\
\text { for potential } \\
\text { confounders } \\
\text { assessed }\end{array}$ \\
\hline $\begin{array}{c}\text { Henning et al., } \\
\text { 2009 [8] }\end{array}$ & Yes & Yes & No & Yes & Yes & Yes & No \\
$\begin{array}{c}\text { Ly et al., 2006 } \\
\text { [13] }\end{array}$ & Yes & Yes & Yes & Yes & Yes & Yes & No \\
$\begin{array}{c}\text { Mulier et al., } \\
\text { 2002 [16] }\end{array}$ & Yes & Yes & Yes & Yes & Yes & Yes & No \\
\hline
\end{tabular}

USPSTF $=$ US Preventive Task Force.

Haenszel formula. Discrete variables were analyzed using risk ratios, whereas continuous variables were analyzed with standard mean difference.

\section{Results}

\section{Hardware Removal}

When considering the proportion of patients undergoing hardware removal, the risk ratio was 0.23 (95\% confidence interval $[\mathrm{CI}], 0.11-0.45 ; \mathrm{p}<0.001)$ indicating more hardware removal for ORIF than fusion (Fig. 2).

\section{Revision Surgery}

For revision surgery, excluding hardware removal, the risk ratio for ORIF was $0.36(95 \% \mathrm{CI}, 0.08-1.59 ; \mathrm{p}=0.18)$ favoring neither (Fig. 3).

\section{Patient Outcome Measures}

Neither ORIF nor fusion was favored when considering patient-reported outcomes (Fig. 4). The standard mean difference was calculated to be $0.50(95 \% \mathrm{CI},-2.13$ to $3.12 ; \mathrm{p}=0.71)$.
Anatomic Reduction

Neither ORIF nor fusion was favored when considering the risk of nonanatomic alignment (Fig. 5). The risk ratio was 1.48 (95\% CI, 0.34-6.38; $\mathrm{p}=0.60)$.

\section{Discussion}

Lisfranc complex injuries are rare. Their diagnosis can be difficult and when missed, patients have poor reported outcomes [2]. Anatomic reduction has been shown to be the primary determining factor in achieving acceptable outcomes [27]. How to best achieve this result remains debatable [19]. RCTs can be difficult to perform in a timely manner given the relatively rare nature of the injury. Previous systematic reviews contained methodological flaws that bring their results into question. Both previous reviews came to the conclusion that further RCTs would be needed to compare the results of these two procedures. No previous study has attempted to perform a meta-analysis comparing ORIF with primary fusion for acute Lisfranc injuries. Our systematic review revealed one currently registered trial comparing ORIF and primary fusion of Lisfranc injuries head to head. No other trials have been published since the studies from 2012. The purpose of this paper was to perform a methodologically sound systematic review and meta-analysis to determine if the present 


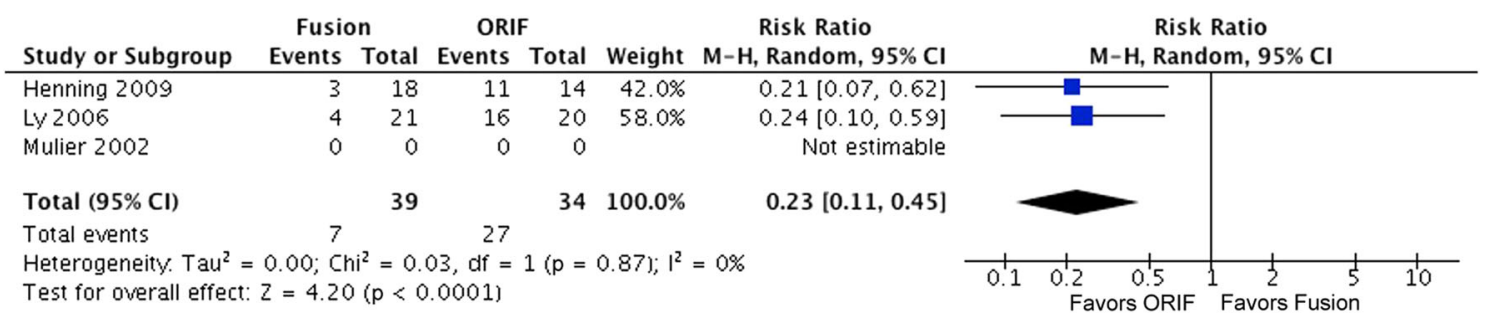

Fig. 2 The forest plot shows the risk ratio for hardware removal. Patients undergoing ORIF are more likely to require removal of hardware. M-H $=$ Mantel-Haenszel; $\mathrm{df}=$ degree of freedom

\begin{tabular}{|c|c|c|c|c|c|c|c|c|c|}
\hline Study or Subgroup & $\begin{array}{l}\text { Fusio } \\
\text { Events }\end{array}$ & Total & $\begin{array}{l}\text { ORI } \\
\text { Events }\end{array}$ & Total & Weight & $\begin{array}{c}\text { Risk Ratio } \\
\text { M-H, Random, 95\% Cl }\end{array}$ & \multicolumn{2}{|c|}{$\begin{array}{c}\text { Risk Ratio } \\
\text { M-H, Random, } 95 \% \mathrm{CI}\end{array}$} & \\
\hline Henning 2009 & 0 & 18 & 1 & 14 & $20.2 \%$ & $0.26[0.01,6.01]$ & & & \\
\hline Ly 2006 & 1 & 21 & 7 & 20 & $43.0 \%$ & $0.14[0.02,1.01]$ & & & \\
\hline Mulier 2002 & 1 & 6 & 2 & 16 & $36.8 \%$ & $1.33[0.15,12.15]$ & & & \\
\hline Total $(95 \% \mathrm{Cl})$ & & 45 & & 50 & $100.0 \%$ & $0.36[0.08,1.59]$ & & & \\
\hline Total events & 2 & & 10 & & & & & & \\
\hline $\begin{array}{l}\text { Heterogeneity. Tau } \\
\text { Test for overall effec }\end{array}$ & $\begin{array}{l}0.29 ; \mathrm{Ch} \\
z=1.35\end{array}$ & $\begin{array}{l}i^{2}=2 \\
(p=0\end{array}$ & $\begin{array}{l}39, \mathrm{df}= \\
181\end{array}$ & $(p=$ & $0.301 ; 1^{2}$ & $=16 \%$ & $\begin{array}{c}0.1 \\
\text { Favors ORIF }\end{array}$ & $10 \frac{10}{\text { Favors Fusion }}$ & 1000 \\
\hline
\end{tabular}

Fig. 3 The forest plot shows the risk ratio for revision surgery (excluding removal of hardware). Neither procedure favors revision surgery. M-H $=$ Mantel-Haenszel; df = degree of freedom.

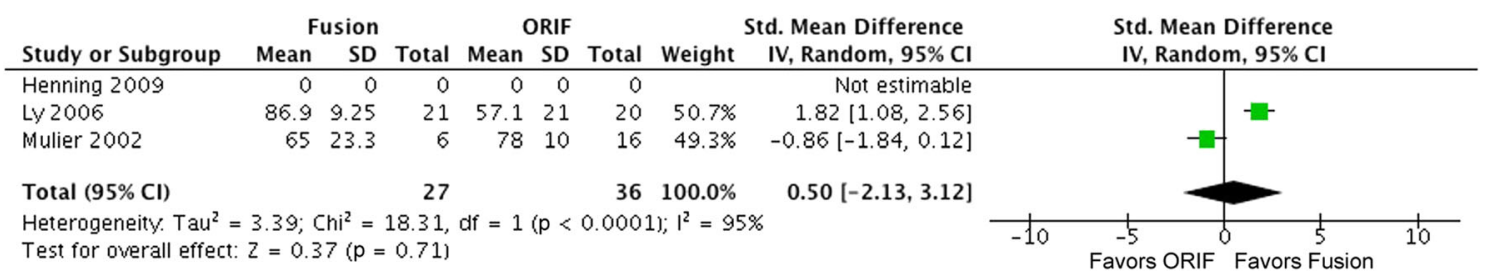

Fig. 4 The forest plot shows the standard mean difference for patient outcome measures (AOFAS and Baltimore Painful Foot System). df = degree of freedom.

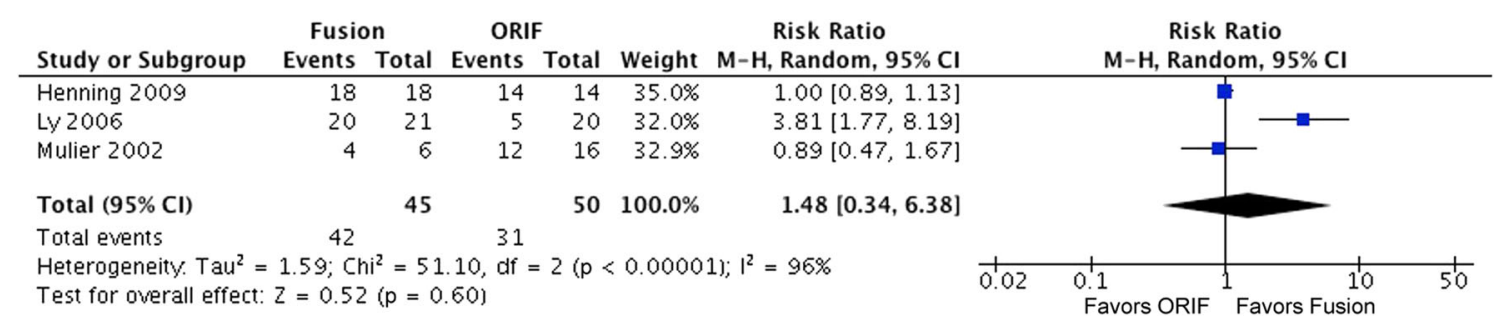

Fig. 5 The forest plot shows the risk ratio for anatomic reduction of Lisfranc complex. M-H = Mantel-Haenszel; df = degree of freedom.

literature demonstrates a clear advantage to either ORIF or primary arthrodesis for Lisfranc injuries. Four surgical outcomes were examined and the only difference found was in the frequency with which patients subsequently underwent removal of hardware with this taking place more often in patients treated with ORIF.

Although our study represents the most comprehensive systematic review and the only meta-analysis related to this topic, there were still potential sources of error. Only three studies within the literature directly compare ORIF with fusion with a total of 101 patients. This low level of recruitment contributed to the large CIs noted for each of our primary outcomes and highlights the need for more highquality comparative trials to build on our current knowledge. High levels of heterogeneity between the studies also demonstrate the difficulty in directly comparing the outcomes from each of these trials. Lisfranc injuries represent a wide range of pathology from purely ligamentous to complex open fracture dislocations. Although no studies included open fractures, each study had slightly different 
inclusion criteria. This will lead to some amount of bias through the comparison of different injury types. Differences in surgical procedures will also contribute to this problem. Most importantly Henning chose to remove the hardware as a standard protocol in his ORIF cohort. Certainly this brings a protocol bias to the positive outcome of increased hardware removal rates in the ORIF group. Even with this built-in removal from Henning, the rates here were similar to those of Ly et al. [13] with CIs of 0.07 to 0.62 and 0.10 to 0.59 , respectively. Also, three patients in the Henning group refused to undergo hardware removal if they were asymptomatic. This would be expected as a likely scenario with a lack of symptoms being a strong reason to deviate from the study protocol. These reasons lessen the concern for the major difference in the hardware removal protocol and allow for the conclusion that the ORIF group undergoes increased removal of hardware.

Each of the individual studies also had design errors that will insert bias into the results of the meta-analysis. Blinding was not well accounted for in any of the studies nor were randomization techniques ideal. This will add to selection bias although this is consistently a problem with surgical outcome studies. Henning's study was underpowered and there was no power analysis done for Mulier's work. There was a significant loss to long-term followup within Henning's paper that weakens their final conclusions. Finally, publication bias may be present in the literature. Our thorough search of the gray literature did not yield any sets of unpublished data. Given the small amount of direct comparative material, it is likely that even small and negative series would add to the available knowledge and the risk of this bias may be low. With only three completed trials, there is no way to examine for publication bias and it must be recognized as a potential confounder. Well-defined comparative trials of similar populations and injury types will help to correct these issues.

The ORIF grouped demonstrated a clear need for increased removal of hardware rates. In considering this positive result, two important factors must be examined. First, Henning et al., as a part of their standard protocol, removed the hardware in 11 of their 14 patients. Three refused stating they were asymptomatic. This protocol bias may have strongly influenced their removal rates. Ly et al. [13] had similar removal rates without such a standard protocol. Second, only two of the three studies reported on hardware removal. The need for this remains debatable. Certainly this is an extremely important variable for patients to understand given the large impact a second, potentially unnecessary, surgery would have on their lives. Future studies examining the necessity of hardware removal and the patient impact factor of the procedure will be key in determining the superiority of ORIF versus fusion.

Major revision or repeat surgery would have an even greater impact on a patient's livelihood and satisfaction

Table 3. ORIF outcome measurements

\begin{tabular}{lllll}
\hline Study & Design & Number of patients & Mean outcome measures & Mean followup \\
\hline Ghate et al., 2012 [7] & Case series & 19 & AOFAS 77.5 & 30 months \\
Marin-Pena et al., 2012 [14] & Case series & 32 & AOFAS 91.7 & 14 years \\
Yang et al., 2011 (abstract only) [28] & Case series & 47 & AOFAS 78\% 70 & 28 months \\
Zhu et al., 2011 (abstract only) [30] & Case series & 41 & AOFAS $84.2 \pm 2.8$ & 36 months \\
Llano et al., 2010 (abstract only) [12] & Case series & 20 & AOFAS 85.42 & 39.4 months \\
Zhou et al., 2008 (abstract only) [29] & Case series & 49 & AOFAS 84.2 & Not stated \\
Rajapaske et al., 2006 [21] & Case series & 16 & AOFAS 78.3 & 42.6 months (significant loss) \\
Perugia et al., 2003 [20] & Case series & 42 & AOFAS $81 \pm 13.5$ & 58.4 months \\
Kuo et al., 2000 [9] & Case series & 48 & AOFAS 77 & 52 months (significant loss) \\
Mulier et al., 1997 [17] & Case series & 9 & BPFS 77 & 3 years \\
\hline
\end{tabular}

ORIF = open reduction and internal fixation; AOFAS = American Orthopaedic Foot and Ankle Society painful foot scale; BPFS - Baltimore Painful Foot System.

Table 4. Fusion outcome measurements

\begin{tabular}{lllll}
\hline Study & Design & Patients & Mean outcome measure & Mean followup (months) \\
\hline Reinhardt et al., 2012 [23] & Case series & 25 & AOFAS 81 & 42 \\
Lin et al., 2000 [11] & Case series & 16 & AOFAS 70 & 36 \\
Saxena, 1997 [24] & Case series & 7 & AOFAS 86.4 & 24 \\
Mulier et al., 1997 [17] & Case series & 9 & BPFS 59 & 3
\end{tabular}

AOFAS = American Orthopaedic Foot and Ankle Society painful foot scale; BPFS = Baltimore Painful Foot System. 
than simple removal of hardware. A clearly superior and definitive procedure in this category would strongly influence a surgeon to complete one operation over the other. We were unable to demonstrate this difference with a metaanalysis of the currently available patients. RCTs of adequate quality and followup need to be performed to capture this outcome.

Validated patient outcome scores give us a window into the lives of the patients postoperatively. The use of multiple different scales for patient-defined outcomes makes this variable perhaps the most difficult to interpret. Neither procedure demonstrated an advantage over the other. Stavlas et al. performed a systematic review in 2010 examining the role of reduction and internal fixation in the management of Lisfranc injuries [26]. They pooled the results of all reported AOFAS scores within the literature and found the mean score to be 78.1. Since 2010, several case series have been published with mean scores similar or higher than that reported by Stavlas et al. One other comparative trial was found comparing primary ORIF with delayed fusion [22]. This well-designed study compared the results of 22 primary fixations with 22 delayed fusions at a mean of 22 months postinjury. They found higher AOFAS scores in the group fixed primarily compared with the delayed fusion cohort (AOFAS mean 81 versus $72 ; p=0.031$ ). With this currently available body of literature, future studies should include the AOFAS measurement for comparative purposes.

Anatomic reduction of the Lisfranc complex has been clearly demonstrated as the most important factor in determining patient outcomes after these injuries. Our inability to show a clearly superior procedure between ORIF and fusion may stem from the fact that reduction rates appear to be similar between the two operations. We were unable to demonstrate a difference in reduction rates. High-quality radiography, either plain film or CT, and clear definitions of reduction are needed for future work.

The low incidence of Lisfranc injuries makes comparative trials difficult to perform. During our search we discovered many case series that describe outcomes for both ORIF (Table 3) and primary fusion (Table 4). Although this quality of data is not acceptable for inclusion within a meta-analysis, it adds important information to the potential outcomes and body of knowledge surrounding this issue. Previous trials have found advantages to both ORIF and fusion given their patient populations. With only two RCTs and one cohort study it is difficult to eliminate the bias within the population and come to firm conclusions. Unfortunately, no new comparative trials have been performed since the systematic reviews of 2012.

Using the currently available literature, it was asked if ORIF or primary fusion was clearly superior for acute Lisfranc injuries in terms of removal of hardware, revision surgery, patient outcome scores, or anatomic reduction.
Removal of hardware rates appear to be more frequent in patients undergoing ORIF. The need for a second surgery, and the associated negative effect on patient outcomes, must be strongly considered and may lend some surgeons to consider primary fusion as the superior operation. No other differences were established.

When discussing treatment methods with patients, surgeons should consider comorbidities, patient characteristics, fracture pattern, and surgical preferences in their decision-making pathway. Patients undergoing ORIF should be informed of the increased likelihood that they might subsequently undergo removal of hardware compared with a primary fusion. The wide breadth of injury patterns included within the Lisfranc category may introduce too much heterogeneity to conclude that a single procedure is superior in all instances. The results of welldesigned, prospective RCTs will be required to further our knowledge of these treatment modalities.

\section{References}

1. Aitken AP, Poulson D. Dislocation of the tarsometatarsal joint. J Bone Joint Surg Am. 1963;45:246-260.

2. Buzzard BM, Briggs PJ. Surgical management of acute tarsometatarsal fracture dislocation in adult. Clin Orthop Relat Res. 1998;353:125-133.

3. Center for Evidence Based Medicine: Critical appraisal tools. Available at: www.cebm.net/critical-appraisal/. Accessed November 7, 2014.

4. Coetzee JC. Making sense of Lisfranc injuries. Foot Ankle Clin North Am. 2008;13:695-704.

5. Desmond EA, Chou LB. Current concepts review: Lisfranc injuries. Foot Ankle Int. 2006;27:653-660.

6. English TA. Dislocations of the metatarsal bone and adjacent toe. J Bone Joint Surg Br. 1964;46:700-704.

7. Ghate SD, Sistla VM, Nemade V, Vibhute D, Shahane SM, Samant AD. Screw and wire fixation for Lisfranc fracture dislocations. J Orthop Surg. 2012;20:170-175.

8. Henning JA, Jones CB, Sietsema DL, Bohay DR, Anderson JG. Open reduction and internal fixation versus primary arthrodesis for Lisfranc injuries: a prospective randomized study. Foot Ankle Int. 2009;30:913-922.

9. Kuo RS, Tejwani NC, DiGiovanni CW, Holt SK, Benirschke SK, Hansen ST, Sangeorzan BJ. Outcome after open reduction internal fixation of Lisfranc joint injuries. J Bone Joint Surg Am. 2000;82:1609-1618.

10. Lewis C, Mauffrey C, Dickenson E. Open reduction and internal fixation compared with primary arthrodesis of Lisfranc injuries: a systematic review of the literature. Curr Orthop Pract. 2012;23:595-600.

11. Lin SS, Bono CM, Treuting R, Shereff M. Limited intertarsal arthrodesis using bone grafting and pin fixation. Foot Ankle Int. 2000;21:742-748.

12. Llano IU, Sobron OS, Celada AC, Sanchez IG, Sanchez IG, de los Mozos JL. [Lisfranc fracture dislocation] [in Spanish]. Gac Med Bilboa. 2010;107:59-63.

13. Ly TV, Coetzee JC. Treatment of primarily ligamentous Lisfranc joint injuries: primary arthrodesis compared with open reduction and internal fixation. J Bone Joint Surg Am. 2006;88:514-520. 
14. Marin-Pena OR, Recio FV, Gomez TS, Garijo RL. Fourteen years follow up after Lisfranc fracture-dislocation: functional and radiological results. Inj Int J Care Injured. 2012;43:79-82.

15. Moher D, Liberati A, Tetzlaff J, Altman DG, The PRISMA Group. Preferred reporting items for systematic reviews and meta-analyses: the PRISMA statement. PLoS Med. 2009;6:1-6.

16. Mulier T, Reynders P, Dereymaeker G, Broos P. Severe Lisfranc injuries: primary arthrodesis or ORIF? Foot Ankle Int. 2002;23:902-905.

17. Mulier T, Reynders P, Sioen W, van den Bergh J, Reymaeker G, Reynaert, Broos P. The treatment of Lisfranc injuries. Acta Orthop Belg. 1997;63:82-90.

18. Myerson MS, Fisher RT, Burgess AR, Kenzora JE. Fracture dislocations of the tarsometatarsal joints: end results correlated with pathology and treatment. Foot Ankle. 1986;9:225-242.

19. Panagakos P, Patel K, Gonzalez CN. Lisfranc arthrodesis. Clin Podiatr Med Surg. 2012;29:51-66.

20. Perugia D, Basile A, Battaglia A, Stopponi M, de Simeonibus AU. Fracture dislocations of Lisfranc's joint treated with closed reduction and percutaneous fixation. Int Orthop. 2003;27:30-35.

21. Rajapaske B, Edwards A, Hong T. A single surgeon's experience of the treatment of Lisfranc joint injuries. Injury. 2006;37:914921.

22. Rammelt S, Schneiders W, Schikore H, Holch M, Heineck J, Zwipp H. Primary open reduction and fixation compared with delayed corrective arthrodesis in the treatment of tarsometatarsal (Lisfranc) fracture dislocation. $J$ Bone Joint Surg $\mathrm{Br}$. 2008;90:1499-1506.
23. Reinhardt KR, Oh LS, Schottel P, Roberts MM, Levine D. Treatment of fracture-dislocations with primary partial arthrodesis. Foot Ankle Int. 2012;33:50-56.

24. Saxena A. Trauma to Lisfranc's joint: an algorithmic approach. Lower Extremity. 1997;4:87-96.

25. Sheibani-Rad S, Coetzee JC, Giveans MR, DiGiovanni C. Arthrodesis vs ORIF for Lisfranc fractures. Orthopedics. 2012;35:868-873.

26. Stavlas P, Roberts CS, Xypnitos FN, Giannoudis PV. The role of reduction and internal fixation of Lisfranc fracture-dislocations: a systematic review of the literature. Int Orthop. 2010;34:1083-1091.

27. Teng AL, Pinzur MS, Lomasney L, Mahoney L, Havey R. Functional outcome following anatomic restoration of tarsalmetatarsal fracture dislocation. Foot Ankle Int. 2002;23:922-926.

28. Yang Y, Yu G, Zhou J, Li B, Li H, Zhu X. [Treatment of occult Lisfranc injury with open reduction and internal fixation] [in Chinese]. Zhongguo Xiи Fu Chong Jian Wai Ke Za Zhi. 2011;25:785-788.

29. Zhou Y, Wu X, Zhang Y. Surgical treatment of Lisfranc fracturedislocations by reconstruction of three column with Kirschner wire fixation. China J Orthop Trauma. 2008;21:539-540.

30. Zhu H, Zhao H, Yuan F, Yu G. Effective analysis of open reduction and internal fixation for the treatment of acute Lisfranc joint injury. China J Orthop Trauma. 2011;24:922-925. 